Indian J. Anim. Hlth. (2019), 58(1) : 115-120

DOI: https://doi.org/10.36062/ijah.58.1.2019.115-120

\title{
A RETROSPECTIVE STUDY ON THE SEROPREVALENCE OF BRUCELLOSIS IN TIBET
}

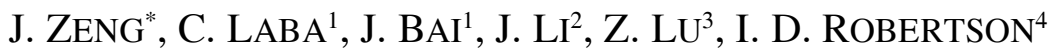 \\ Department of Veterinary, Tibet Livestock Research Institute \\ Tibet Academy of Agriculture and Animal Science \\ Lhasa, Tibet, China
}

\begin{abstract}
A retrospective study on the seroprevalence of brucellosis in livestock in Tibet covering almost 4 decades and in humans for the past decade was undertaken. In the $20^{\text {th }}$ century, the prevalence of brucellosis in livestock was greatly reduced through a mass vaccination program. However, early in the $21^{\text {st }}$ century, significant differences were found in the spatial and temporal distribution of brucellosis in both livestock and humans $(p<0.05)$. In the period from 2011 to 2013 , there was a positive correlation between the seroprevalence in livestock and that of humans $(r=0.93)$. Brucellosis was more common in livestock in the spring/summer seasons when parturition occurred. The findings from this study can be used as baseline epidemiological data for further research on brucellosis in Tibet.
\end{abstract}

Key words: Brucellosis, Retrospective study, Seroprevalence, Tibet

Brucellosis in animals was first reported in Tibet as early as 1931, with evidence of the disease being a severe epidemic by the 1980 s with the prevalence subsequently reducing through the implementation of a vaccination program (Anonymous, 1985). However in the $21^{\text {st }}$ century, evidence of re-emergence of human brucellosis was reported in Tibet (Zhan et al., 2008). Currently, there is little published information about the epidemiology of brucellosis in Tibet, even though the Tibet Centre for Animal Disease Control (TCADC) and the Tibet Centre for Disease Control (TCDC) have been implementing annual serological surveillance programs since 2009. Understanding the epidemiology of a disease plays an important role in developing effective control and prevention programs for that disease. An initial component of this often involves examining and analysing existing historical data. Therefore, the aim of the study reported in this manuscript was to explore the epidemiological characteristics of brucellosis in Tibet through examining historical data from both livestock and humans.

Data from the 1970s and 1980s were collected from articles published in Tibetan

\footnotetext{
* Corresponding Author

1 Tibet Centre for Animal Disease Control, Lhasa, Tibet, China

2 Tibet Centre for Human Disease Control, Lhasa, Tibet, China

3 Chengdu Vocational College of Agricultural Science and Technology, Chengdu, Sichuan, China

4 School of Veterinary Medicine, Murdoch University, Perth, Western Australia, Australia
} 
journals and books. The data set of recent cases/diagnoses of brucellosis in yaks, cattle, sheep, goats and pigs from 2009 to 2015 in Tibet was obtained from the TCADC. The disease (seropositivity) was based on a positive reaction to the rose-bengal plate test (RBPT). Data on human cases of brucellosis from 2011 to 2013 in Tibet were obtained from the TCDC. These data provided information on the total number of humans tested and the number positive to the tube (serum) agglutination test, the standard test for the serological diagnosis of brucellosis (Gupte and Kaur, 2015).

An Excel (Microsoft Office Excel 2010) spreadsheet was developed for the data and statistical tests were undertaken using SPSS Statistics for Windows (Version 21.0, IBM Corp., Armonk, New York, USA) and R (R Development Core Team, 2013).

From 1979 to 1985 , blanket vaccination of livestock was undertaken compulsorily in all 64 counties of Tibet. More than 19 million head of livestock were vaccinated, representing $91 \%$ of the total population. The results available from the four prefectures (prefectural cities) of Lhasa, Shannan, Shigatse and Chamdo sampled showed a considerable reduction in the seroprevalence from $13.2 \%(95 \% \mathrm{CI}: 13.0,13.4)$ prior to 1979 to $0.4 \%$ (95\%CI: $0.3,0.4)$ in 1985 $\left(\chi^{2}=6195.72, \mathrm{df}=1, \mathrm{p}<0.001\right)(\operatorname{Pan}, 1992)$.

Between 2009 and 2015, 32,440 yaks, cattle, sheep, goats and pigs were sampled and tested for brucellosis with 265 positive (0.82\%; 95\%CI: 0.7, 0.9). Evidence of infection (seropositive animals) was found in all prefectures (Table 1). The prevalence was significantly different between the seven prefectures $\left(\chi^{2}=75.73, \mathrm{df}=6\right.$, $\mathrm{p}<0.001)$. No seropositive animals were detected in 2009 and 2013 (Table 2). Overall there was a significant difference in the seroprevalence between years $\left(\chi^{2}=302.44, \quad \mathrm{df}=6, \quad \mathrm{p}<0.001\right)$. The seroprevalences in 2010, 2011, 2012 and 2014 were significantly higher than that in

Table 1. Seroprevalence of brucellosis in yaks, cattle, sheep, goats and pigs at the prefecture-level in Tibet (2009-2015)

\begin{tabular}{lcccc}
\hline Prefecture & $\begin{array}{c}\text { No. } \\
\text { positive }\end{array}$ & $\begin{array}{c}\text { No. } \\
\text { tested/sampled }\end{array}$ & $\begin{array}{c}\text { Prevalence } \\
(\mathbf{9 5 \% C I )}\end{array}$ & $\begin{array}{c}\text { Odd ratios } \\
(\mathbf{9 5 \%} \% \mathbf{C})\end{array}$ \\
\hline Ngari & 66 & 3580 & $1.84(1.4,2.3)$ & $8.71(4.47,16.97)$ \\
Chamdo & 44 & 3863 & $1.14(0.8,1.5)$ & $5.38(2.71,10.71)$ \\
Lhasa & 41 & 5864 & $0.70(0.5,0.9)$ & $3.31(1.65,6.60)$ \\
Shannan & 40 & 5694 & $0.70(0.5,1.0)$ & $3.32(1.66,6.65)$ \\
Nagqu & 34 & 4386 & $0.78(0.5,1.1)$ & $3.66(1.81,7.43)$ \\
Shigatse & 30 & 4326 & $0.69(0.5,1.0)$ & $3.28(1.60,6.71)$ \\
Nyingchi & 10 & 4727 & $0.21(0.1,0.4)$ & 1.0 \\
\hline
\end{tabular}




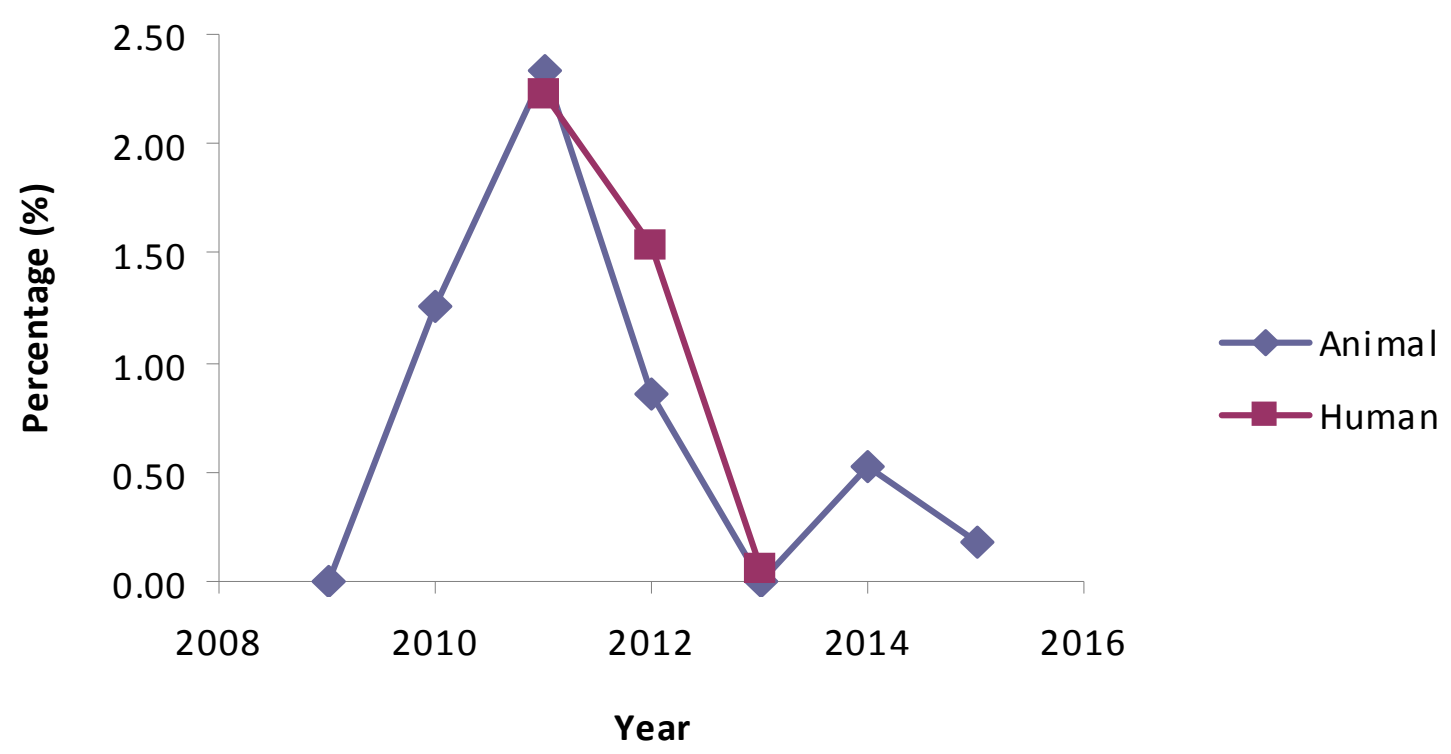

Fig. 1. Annual seroprevalence of animal and human brucellosis in Tibet (2009-2015)

2015. Overall the seroprevalence was significantly higher in the spring / summer seasons $(1.32 \%, 95 \% \mathrm{CI}: 1.1,1.5)$ than in autumn / winter seasons $(0.41 \%, 95 \% \mathrm{CI}$ : $0.3,0.5)\left(\mathrm{p}<0.001, \chi^{2}=64.95, \mathrm{df}=1\right)$.

A total of 7421 humans were tested for brucellosis from 2011 to 2013 with 62 positives $(0.84 \%$; $95 \% \mathrm{CI} 0.6,1.1)$ (no data were available for other years). Initially the seroprevalence of brucellosis in humans was different between prefectures $\left(p=0.038, \chi^{2}=11.80, d f=5\right)$ (Table 3). The seroprevalence in humans varied significantly over the three years of sampling $\left(\mathrm{p}<0.001, \chi^{2}=66.47, \mathrm{df}=2\right)$, decreasing from $2.23 \%$ (95\% CI: $1.4,3.4$ ) in 2011 to $0.05 \%$ (95\% CI: $0.0,0.2)$ in 2013.

The seroprevalence in livestock from the different prefectures was not significantly correlated with the seroprevalence in humans from those prefectures $(r=0.31, p$ $=0.556)$. However, there was a positive, although not significant, correlation in the overall seroprevalence of animals and humans for the years from 2011 and 2013 $(\mathrm{r}=0.93, \mathrm{p}=0.233)$ (Fig 1).

This is the first study examining the epidemiological characteristics of brucellosis in animals and humans in Tibet using historical data.

During the 1970s and early 1980s, three Brucella species (B. melitensis, B. abortus and $B$. suis) were isolated and identified in Tibet. Through the use of $B$. suis $\mathrm{S} 2$ vaccine and $B$. melitensis M5 vaccine the seroprevalence in animals decreased significantly (Pan, 1992). 
Table 2. Seroprevalence of animal brucellosis during the period of 2009 to 2015

\begin{tabular}{lcccc}
\hline Year & $\begin{array}{c}\text { No. } \\
\text { positive }\end{array}$ & $\begin{array}{c}\text { No. } \\
\text { sampled }\end{array}$ & $\begin{array}{c}\text { Seroprevalence (\%) } \\
(\mathbf{9 5 \%} \text { CI) }\end{array}$ & $\begin{array}{c}\text { OR } \\
(\mathbf{9 5 \%} \text { CI) }\end{array}$ \\
\hline 2009 & 0 & 2030 & $0.00(0.0,0.2)$ & - \\
2010 & 36 & 2848 & $1.26(0.9,1.7)$ & $7.28(4.08,12.98)$ \\
2011 & 163 & 6957 & $2.34(2.0,2.7)$ & $13.64(8.27,22.50)$ \\
2012 & 17 & 2000 & $0.85(0.5,1.4)$ & $4.87(2.48,9.56)$ \\
2013 & 0 & 2780 & $0.00(0.0,0.1)$ & - \\
2014 & 32 & 6142 & $0.52(0.4,0.7)$ & $2.98(1.65,5.37)$ \\
2015 & 17 & 9683 & $0.18(0.1,0.3)$ & 1.0 \\
\hline
\end{tabular}

The overall seroprevalence of brucellosis in animals from 2009 to 2015 was $0.82 \%$, which was significantly higher than the recommended level of animal brucellosis in China (0.09-0.28\%) (Wang et al., 2010). In the 1990s, the disease was primarily restricted to the main pastoral areas of China, such as Tibet and Inner Mongolia, however with the rapid development of animal husbandry in inland China it extended into more farming-pastoral and farming regions, including Helongiiang and Shanxi provinces (Wang et al., 2010). Currently, the disease results in regular epidemics in the northern, north-eastern and north-western regions of China (Li et al., 2013).

This study demonstrated evidence of infection in animals in all prefectures of Tibet. The highest prevalence was reported in Ngari, which is an exclusively pastoral area with yaks, sheep and goats being the dominant livestock present. The lowest prevalence was reported in Nyingchi where an agro-forestry-pastoral mixed production system is adopted. The number of livestock in Ngari is more than three times that found in Nyingchi (Duoji and Yang, 2014). A similar epidemiological study in Bhutan for foot and mouth disease demonstrated that the seroprevalence in yaks increased with increasing animal population (Dukpa, 2011) due to more frequent livestock movements and a greater chance of contact of naïve animals with infected animals.

In this study, no infected animals were detected in 2009 and 2013. This could be due to the time of sample collection as the calving season of yaks and sheep in Tibet occurs mainly in April and May (spring/ summer). Surveillance in these two years was conducted during summer and autumn when few animals calves/lambs resulting in less opportunity for the spread of infection which is associated with abortion and parturition (Al-Rawahi, 2014). The highest seroprevalence was observed in 2011 (2.34\%, 95\%CI: 2.0, 2.7). A study by $\mathrm{Li}$ (2013) demonstrated an increase in seropositivity from 2009, reaching a peak of $7.8 \%$ through 2011 in Tongliao district 
Table 3. Seroprevalence of brucellosis in humans at the prefecture-level in Tibet (2011- 2013)

\begin{tabular}{lcccc}
\hline Prefecture & $\begin{array}{c}\text { Number } \\
\text { positive }\end{array}$ & $\begin{array}{c}\text { Total } \\
\text { number }\end{array}$ & $\begin{array}{c}\text { Prevalence (\%) } \\
(\mathbf{9 5 \%} \text { CI) }\end{array}$ & $\begin{array}{c}\text { OR } \\
(\mathbf{9 5 \%} \text { CI) }\end{array}$ \\
\hline Chamdo & 1 & 71 & $1.41(0.0,7.6)$ & $3.05(0.34,27.66)$ \\
Shannan & 27 & 2347 & $1.15(0.8,1.7)$ & $2.48(0.87,7.12)$ \\
Lhasa & 25 & 2537 & $0.99(0.6,1.5)$ & $2.12(0.74,6.12)$ \\
Nagqu & 5 & 960 & $0.52(0.2,1.2)$ & $1.12(0.30,4.18)$ \\
Ngari & 4 & 858 & $0.47(0.1,1.2)$ & 1.0 \\
Shigatse & 0 & 648 & $0.00(0.0,0.6)$ & - \\
\hline
\end{tabular}

of Inner Mongolia (Li, 2013). The seroprevalence in humans followed a similar pattern to that of animals with a rapid increase in the number of new cases from 2003 (6448) to 2011 (38151) in China (Liu et al., 2014). The movement of infected livestock is likely linked with the occurrence and spread of brucellosis (Zamri-Saad and Kamarudin, 2016). In Tibet, the introduction of new breeds, including Jersey and Holstein cattle, to improve the genetics and productivity of the local breeds (Se et al., 2010), could have resulted in increased risk of transmission if the introduced animals were infected and then distributed into susceptible herds/flocks.

This study found a significantly higher seroprevalence during the spring/summer seasons than in the autumn/winter seasons due to coinciding with the calving/lambing/ kidding season $(p<0.001)$. Other reports have similarly shown that higher seroprevalences mainly occur during the spring/summer periods from March to June (Zhu, 2013).
Although, surprisingly, there was no significant correlation in the spatial distribution of animal and human seroprevalence, there was a positive temporal correlation. This latter finding was expected given that infection in humans arises through contact with infected animals or their products (Corbel, 1997).

In conclusion, this study explored the epidemiological characteristics of brucellosis in Tibet over the last four decades and the findings provide valuable information for decision-makers when developing a program to control the disease locally.

\section{ACKNOWLEDGEMENTS}

The authors would like to express their thanks to Tibet Centre for Animal Disease Control and Tibet Centre for Human disease Control for data supply.

Conflict of interest: None to declare 


\section{REFERENCES}

Al-Rawahi A, 2014. The Epidemiology of brucellosis in the Sultanate of Oman. PhD Dissertation. Murdoch Universtiy, Murdoch-6150, Perth, Australia

Anonymous, 1985. Comments of preventing brucellosis in Tibet. Tibetan J Sci Technol, 4: $47-49$

Corbel MJ, 1997. Brucellosis: An overview. Emerg Infect Dis, 3: 213-221

Dukpa KZ, 2011. The epidemiology of footand-mouth disease in the Kingdom of Bhutan. PhD Dissertation. Murdoch University, Murdoch-6150, Perth, Australia

Duoji Z and Yang YM, 2014. Tibet Statistic Yearbook. China Statistices Press, Beijing, CHN

Gupte $S$ and Kaur T, 2015. Diagnosis of human brucellosis. J Trop Dis, 4: 185

Li XY, 2013. Epidemiology survey and preliminary study on the infection mechanism of cow brucellosis in Tongliao district of Inner Mongolia. PhD Dissertation. Jilin University, Chaoyang130012, Changchun, China

Li YJ, Li XL, Liang S, Fang LQ and Cao WC, 2013. Epidemiological features and risk factors associated with the spatial and temporal distribution of human brucellosis in China. BMC Infect Dis, 13(1): 1

Liu Q, Cao L and Zhu XQ, 2014. Major emerging and re-emerging zoonoses in China: A matter of Global health and socioeconomic development for 1.3 Billion. Int J Infect Dis, 25: 65-72
Pan ZF, 1992. Review of Prevention and Cure of Brucellosis in Tibet. In: Veterinary Section of Tibet Animal Husbandry and Veterinary Collection (Tibetan Association of Animal Husbandry and Veterinary, eds). Tibetan Association of Animal Husbandry and Veterinary, Lhasa (China), pp229-232

R Development Core Team, 2013. R: A Language and Environment for Statistical Computing. R Foundation for Statistical Computing, Vienna, Austria. Available in http://www.r-project.org.[19 Nov, 2015]

Se Z, Ao SM, Luosang J and Pingcuo Z, 2010. Achievements of improved cattle in Tibet. Tibetan J Sci Technol, 1: 48-51

Wang GM, Chi LJ, Ma SC, Su ZH, Chen Y et al., 2010. Epidemic characteristics and cause of brucellosis in livestock in China. Chinese J Anim Hlth Inspec, 27: 62-63

Zamri-Saad M and Kamarudin MI, 2016. Control of animal brucellosis: The Malaysian experience. Asian Pac J Trop Med, 9(12): 1136-1140

Zhan D, Ruan SL, Gongsang Q and Cui BY, 2008. Epidemiological analysis of brucellosis in Naqu and Qamdo, Tibet in 2006. Dis Surveill, 23(5): 285-286

Zhu SP, 2013. Human and animals brucellosis epidemic space and time trend analysis and their correlation study. MSc Dissertation. Northwest University for Nationalities, Chengguan-730030, Lanzhou, China 